\title{
AETIOLOGY AND SEVERITY OF GINGIVAL RECESSION AMONG YOUNG INDIVIDUALS IN BELGAUM DISTRICT IN INDIA
}

V. Dodwad. Aetiology and severity of gingival recession among young individuals in Belgaum district in India. Annal Dent Univ Malaya 2001; 8: 1-6.

\section{ABSTRACT}

Gingival recession is used to characterize the apical shift of marginal gingiva from its normal position on the crown of the tooth to the levels on the root surface beyond the cemento-enamel junction. In this study, the sample consisted of 1200 subjects in the district of Belgaum, India. They all belonged to the age group between 15 to 24 years. The subjects were examined for etiology and severity of different grades of gingival recession, utilizing the classification of P.D. Miller Jr. Clinical examination involved assessment of gingival recession, plaque, gingivitis, frenal attachment and frenal pull, trauma from occlusion, presence of calculus, adequacy of attached gingiva and oral hygiene habits. Data collected during the epidemiological survey were analyzed. It was interesting to find out from the above data that when considered in totality, the severity of gingival recession is a result of multifactorial etiology.

Key words: Gingival recession, severity, etiology, young adults.

\section{INTRODUCTION}

The Mucogingival complex consists of free and attached gingiva, mucogingival junction and the alveolar mucosa(1). An adequate mucogingival complex, in which the mucogingival tissues can sustain their biomorphologic integrity and maintain an enduring attachment to the teeth and the underlying soft tissue, is always essential. When a mucogingival problem occurs, there are basically two ways in which it presents itself:

1. As a close disruption of the mucogingival complex resulting in pocket formation.

2. As an open disruption of the mucogingival complex resulting in gingival clefts and gingival recession.

Gingival recession is a term used to characterize the apical shift of the marginal gingiva from its normal position on the crown of the tooth to the levels on the root surface beyond the cemento- enamel junction (1). The etiological factor of great importance is tooth malpositioning followed by faulty or excessive toothbrushing (2). Bacterial plaque is of equal importance in the etiology of recession. There has not been a standardized and consistent adherence to a given classification by various authors in their conclusion
Original Article

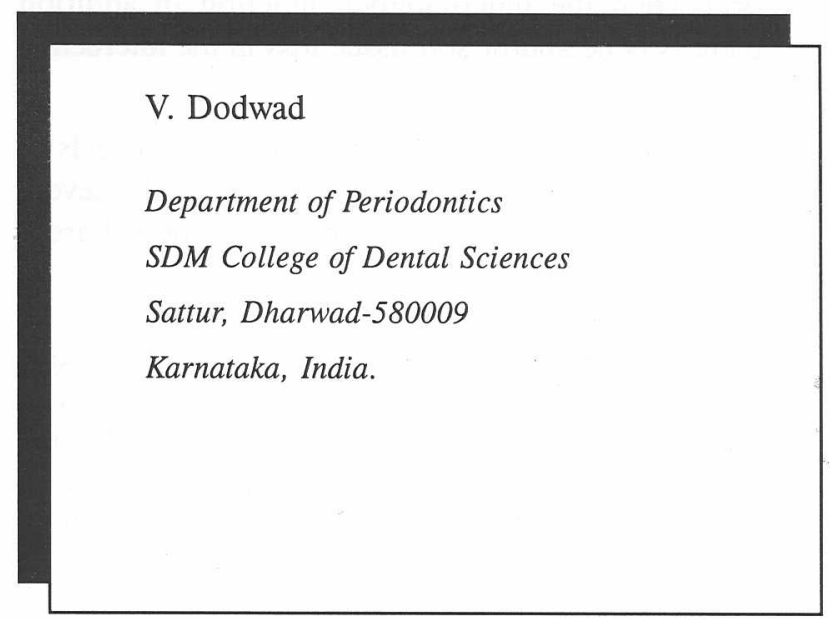

regarding various aspects of gingival recession. Hence there appears to be a need for further study of possible causative factors and severity of gingival recession in young individuals based on approved classification(3), which would not only help in diagnosis but also in the determination of prognosis. The present investigation has been designed to determine the severity and etiology of different grades of gingival recession among young individuals between 15 to 24 years of age groups in Belgaum district in India.

\section{MATERIALS AND METHODS}

An epidemiological study was undertaken to find out the severity and etiology of gingival recession among young individuals in Belgaum district, India. A random sampling technique was used in the study. One thousand two hundred subjects with gingival recession in the age group between 15 to 24 years were examined. In order to collect the data, an interview cum dental examination with the help of a proforma prepared for the study was carried out. Each subject was examined on an ordinary chair, under adequate natural light, using a mouth mirror, explorer and Williams's graduated periodontal probe. Examination of teeth in all the quadrants was done in uniform fashion. Only one investigator examined all the subjects, to avoid interexaminer variability.

Examinations of each subject took approximately 56 minutes and it was carried out in the following sequeale. Personal history of each subject regarding oral hygiene habits like type of toothbrush, frequency of brushing and method of brushing was taken into consideration. Gingival recession was recorded according to the P.D. Miller Jr. classification of marginal tissue recession (3).

Class I-Marginal tissue recession without extending to the mucogingival junction without loss of bone or soft tissue in the interdental areas. 
Class II - Marginal tissue recession that extends to or beyond the mucogingival junction without loss of bone or soft tissue in the interdental areas.

Class III- Marginal tissue recession that extends to or beyond the mucogingival junction in addition there is bone and/or soft tissue loss in the interdental areas.

Class IV- Marginal tissue recession that extends to or beyond the mucogingival junction with severe bone and/or soft tissue loss in the interdental areas and /or severe tooth malposition.

The area was then evaluated by assessing the plaque and calculus accumulation on each tooth. Plaque was scored in a range from 0-3 using plaque index of Silness and Loe (4). Gingival inflammation was assessed by using the gingival index by Loe and Silness (5). Further, the tooth malalignment was observed by viewing the teeth from occlusal plane and position of each tooth was classified according to its relation to the regular curve of the arch either correctly positioned or outstanding (labially placed) and instanding (lingually placed) in all subjects, the upper and lower frenum was examined for the site of attachment and presence of frenal pull. Four types of frenal attachment were considered, according to Placek and co-workers classification (6). Tension test was carried out to confirm the adequacy of the width of the attached gingiva (7). Trauma from occlusion was assessed by checking mobility of each tooth. Fremitus test was done on both upper and lower teeth $(8,9)$.

The data was subjected for statistical analysis by using chi square test.

\section{RESULTS}

Out of the 1200 subjects showing gingival recession 804 subjects $(67 \%)$ were males and $396(33 \%)$ were female
(Fig. 1). Majority (86.16\%) of the subjects showed grade I gingival recession and lesser number of subjects, $11.83 \%, 1.83 \%$ and $0.6 \%$ showed grade II, grade III and grade IV gingival recession respectively. About $86.67 \%$ of subjects showed gingival recession in mandibular anterior region (Fig. 2).

Horizontal brushing method and medium type of toothbrush was seen to be more injurious to marginal gingiva leading to gingival recession. Statistical analysis revealed that, these differences between type of brush, brushing method. and gingival recession were significant $\left(\chi^{2}=29.066, \mathrm{DF}=4, \mathrm{p}<.001\right)$. Subjects who brushed once daily showed more gingival recession than those subjects who brushed twice daily or more (Fig. 3). This observation was found to be statistically significant. ( $\chi^{2}$ $=87.864, \mathrm{DF}=2, \mathrm{p}<.001)$. Plaque and gingival inflammation appeared to be the most common precipitating etiological factor (Fig. 4). The association between plaque and gingival inflammation and gingival recession was found to be statistically significant $\left(\chi^{2}=\right.$ $27.296, \mathrm{DF}=6, \mathrm{p}<.001$ ). Gingival and papillary type of labial frenal attachment appeared to be always associated with frenal pull and gingival recession. All cases with gingival papillary type of labial frenal attachment and associated frenal pull due to lack of attached gingiva showed gingival recession. About $32.9 \%$ of subjects with decreased width of attached gingiva showed more gingival recession. Malaligned teeth especially labially positioned teeth were more prone to gingival recession in the presence of poor oral hygiene, gingival inflammation and inadequate width of attached gingiva. Analysis revealed that co-relation between tooth position and gingival recession was statistically significant $\left(\chi^{2}=54.246, D F=2\right.$, $\mathrm{p}<.001)$. Trauma from occlusion did not appear to be directly responsible for gingival recession, but only when associated with other local factors such as plaque, calculus, gingival inflammation and malalignement of teeth. Subjects with subgingival calculus $(65.41 \%)$ were

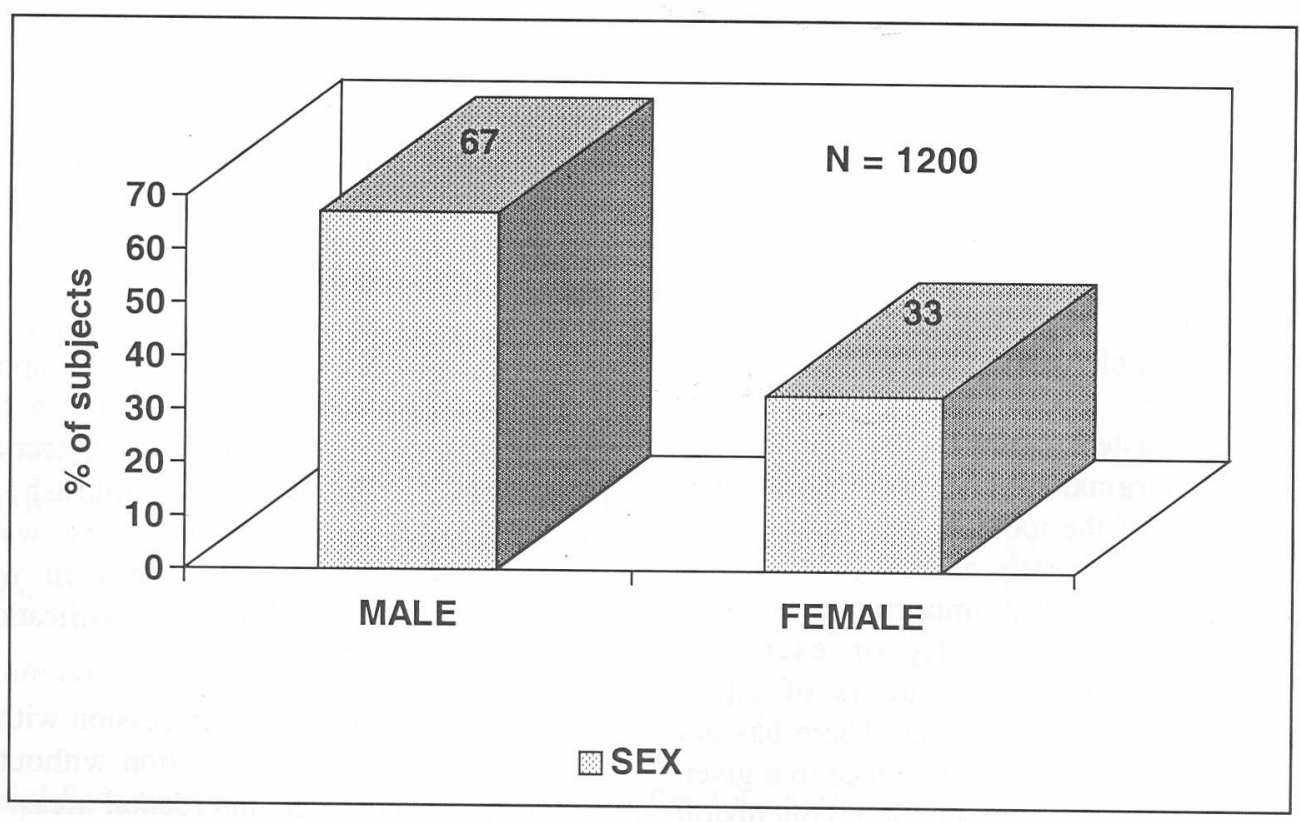

Figure 1: Diagram showing the percentage distribution of male and female subjects with gingival recession. 


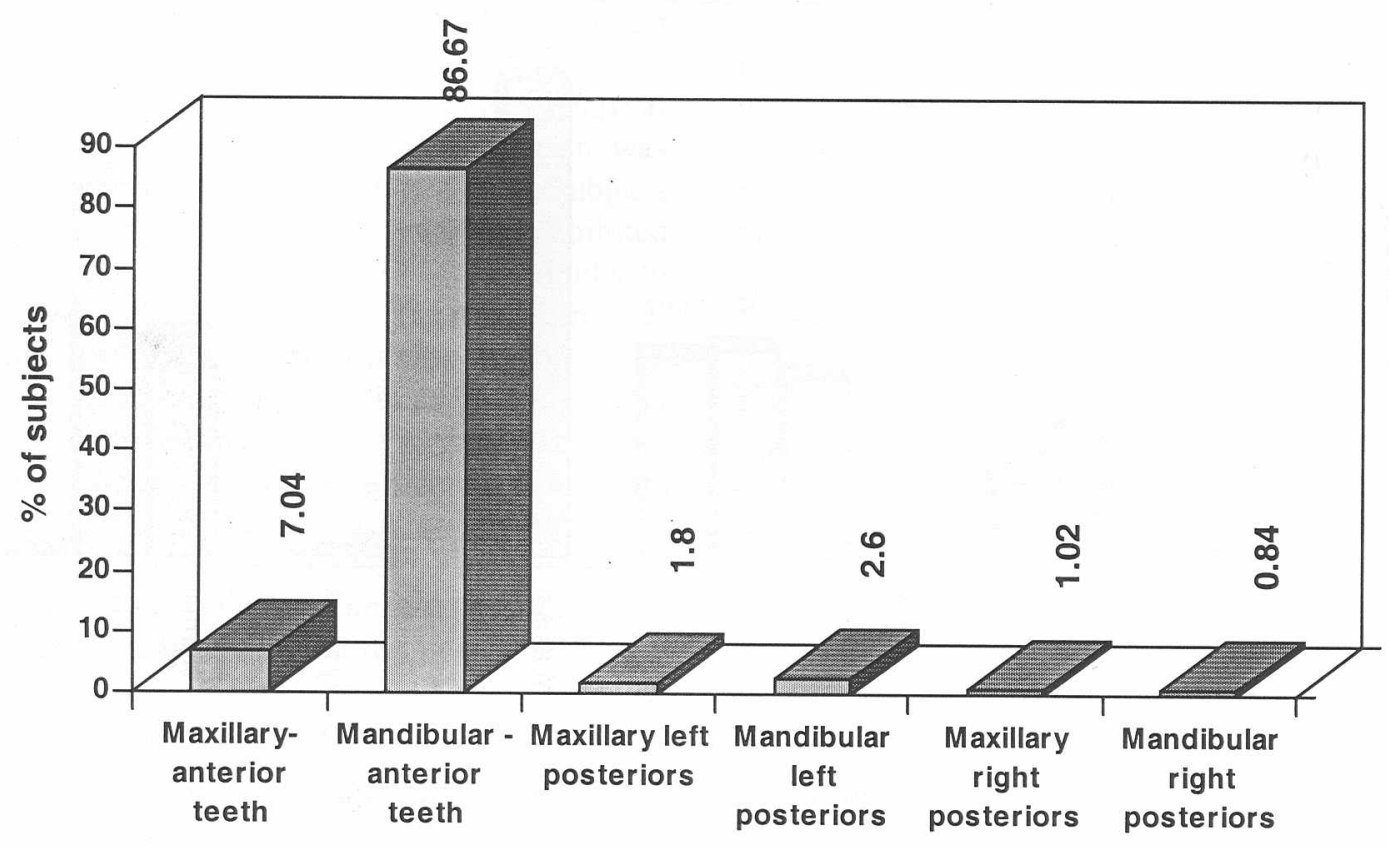

Type of area

Figure 2: Diagram showing percentage of subjects with gingival recession in different areas.

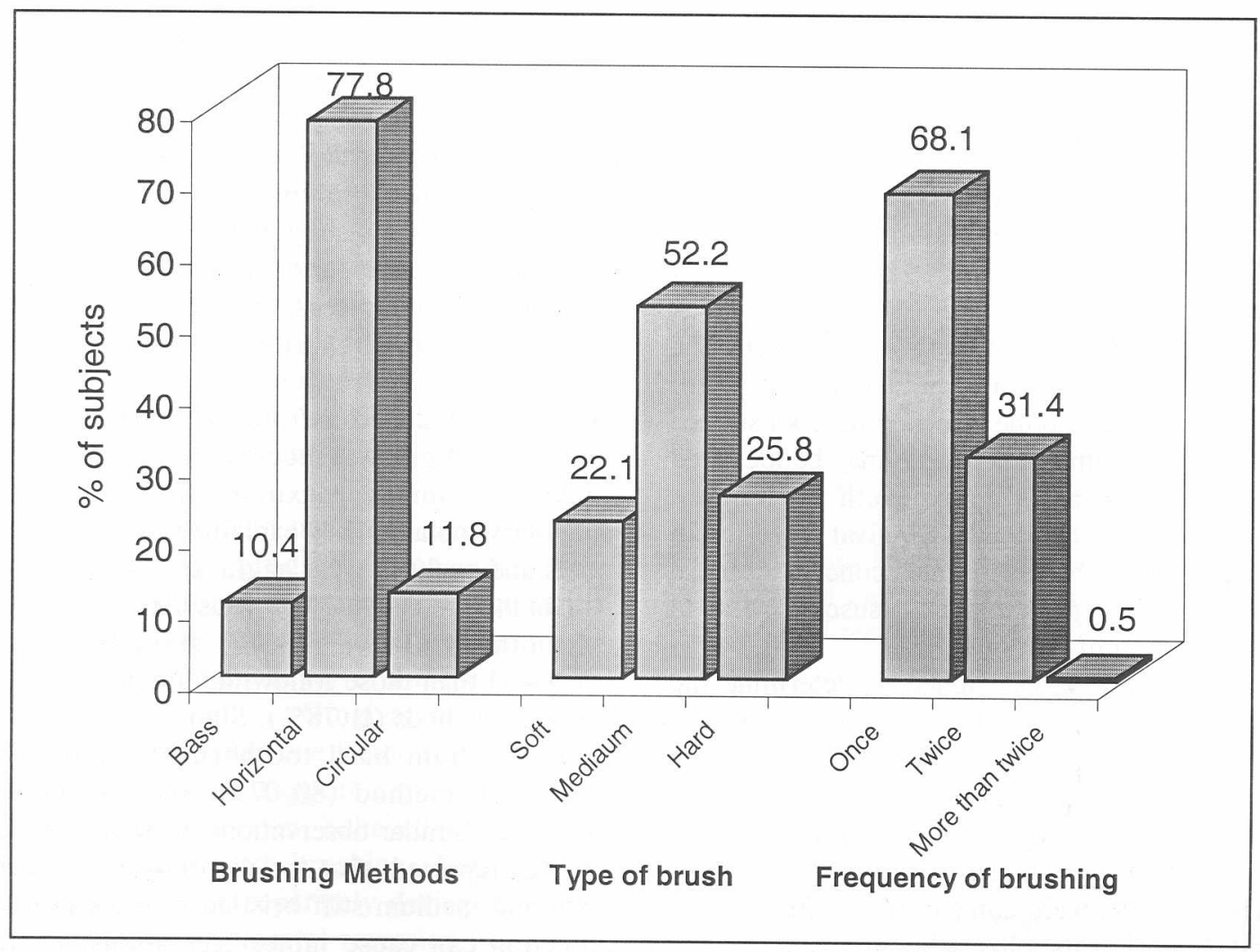

Figure 3: Diagram showing percentage of subjects with different brushing habits. 


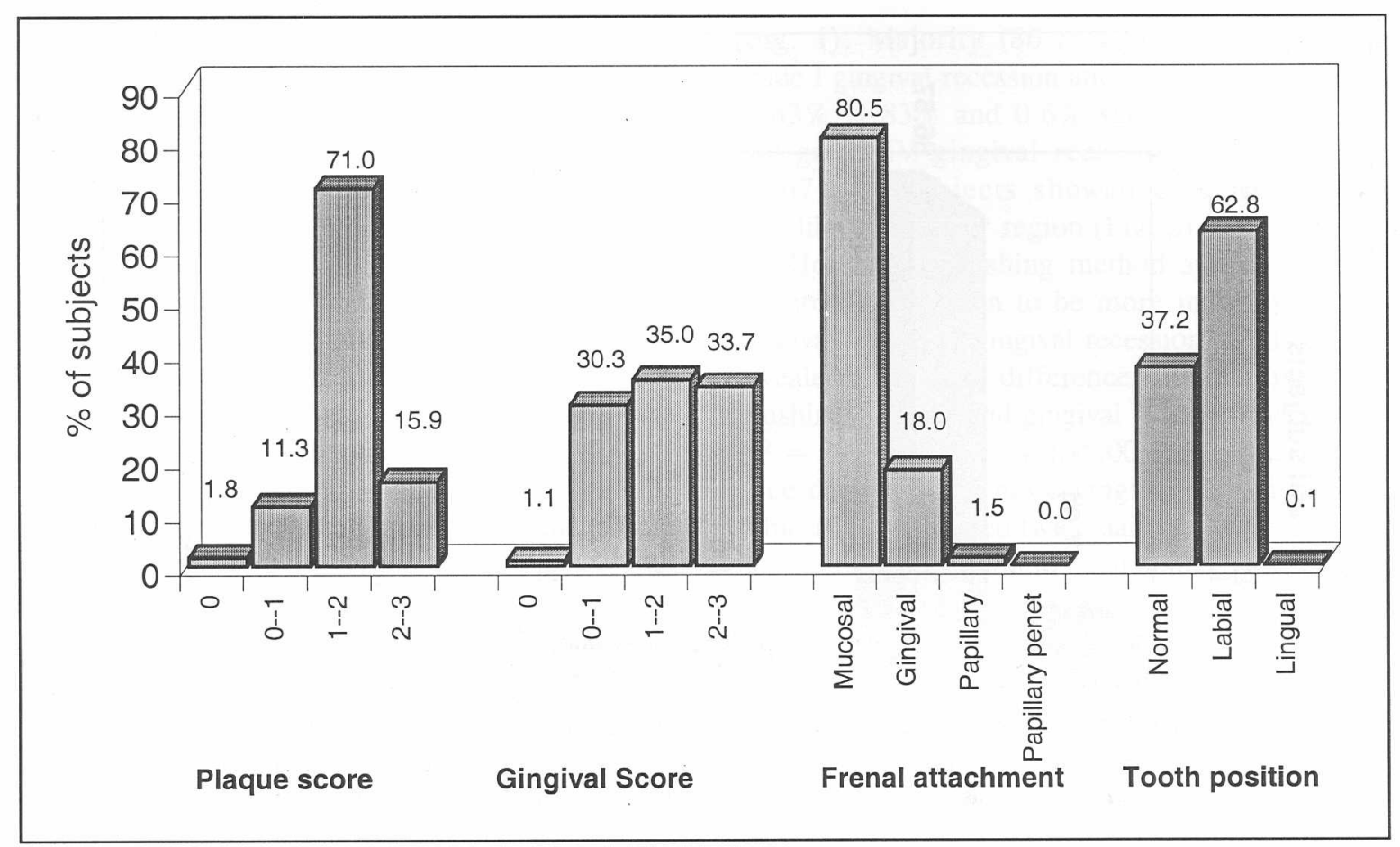

Figure 4: Diagram showing the percentage of subjects with gingival recession associated with different etiological factors.

found to be more associated with gingival recession than those with supra gingival calculus $(34.6 \%)$.

\section{DISCUSSION}

Epidemiology is a useful means of establishing a need for treatment or for preventive intervention. It often deals with co-relations between two or more findings. Various suppositions regarding cause and effect have been based upon these co-relations. However, corelations are not a means of showing cause and effect but only a means of showing a relationship. The exact nature of the relationship remains to be determined in future studies.

Gingival recession is a term used to characterize the apical shift of marginal gingiva from its normal position on the crown of the tooth to the levels on the root surface beyond the cementoenamel junction. It may be localized to a single tooth or a group of teeth or may be generalized throughout the mouth. Gingival recession in relation to anterior teeth is of greater concern because of functional and esthetic anomalies, susceptibility to hypersensitivity and rarely root caries.

The present study was designed to determine the severity and etiology of different grades of gingival recession among young individuals in the age group between 15-24 years in Belgaum district.

Out of the 1200 subjects showing gingival recession, 804 subjects $(67 \%)$ were males and 396 (33\%) were females. These results were comparable to the study of Stoner and Mazdyasma (10) who showed greater recession in males than in females. Ainamo (11) on the other hand found that gingival recession was equally common in both sexes in the 17 years age group. Among the individual teeth affected in the present study, mandibular anterior teeth $(86.7 \%)$ were the most commonly affected teeth with gingival recession followed by maxillary anterior teeth (7.04\%), mandibular left posterior $(2.60 \%)$, maxillary left posteriors $(1.80 \%)$, maxillary right posterior $(1.02 \%)$ and mandibular right posteriors $(0.84 \%)$.

The above findings were in confirmation with the study of Akpata and Jackson (12), who reported gingival recession to be more common in mandibular anterior teeth, which in the young individuals usually are the first to show destructive periodontal disease. With increasing age, periodontal disease as well as buccal recession shows a more generalized pattern.

The results related to the individual teeth involvement differed from the other studies $(13,14)$ which found gingival recession to be more common on maxillary canines, maxillary first premolars and on maxillary molars. They explained that prominent palatal roots and occlusal wear aggravate gingival recession.

In the present study subjects using horizontal method of toothbrushing (77.82\%) showed more gingival recession than those following either Bass $(10.40 \%)$ or circular methods (11.78\%). Similarly subjects who were using medium hard toothbrushes and brushing in horizontal method $(80.07 \%)$ showed more gingival recession. Similar observations made in previous studies $(15,16)$ reported, that too vigorous and forceful use of hard and medium stiff-bristled brushes in a horizontal direction can cause minor lacerations, contusions or abrasions of the gingiva with the resultant cleavage, 
detachment or atrophy of the same as well as resorption of the underlying alveolar plate, which was itself conclusive to gingival recession.

When the association between gingival inflammation, plaque and gingival recession was investigated in the 1200 subjects, $98.2 \%$ of the subjects showed plaque, and $98.9 \%$ of the subjects exhibited gingival inflammation. The present study was similar to other studies $(17,18)$ which showed gingival inflammation to be the most common precipitating etiological factor of gingival recession. They suggested that localized inflammatory process causes the breakdown of connective tissue and proliferation of the epithelium into the site of connective tissue destruction. Proliferation of epithelial cells into the connective tissue brings about a subsidence of the epithelial surface which is manifested clinically as gingival recession. During the study, the association between calculus and gingival recession was considered whereby 665 subjects $(55.41 \%)$ showed presence of calculus. Out of 665 subjects, majority i.e. $435(65.41 \%)$ showed presence of subgingival calculus and 330 subjects $(34.59 \%)$ showed presence of supragingival calculus. These findings coincided with other studies $(18,19)$ which reported that calculus played an important role in the etiology of gingival recession.

In the present study, when the association between the adequacy of the attached gingiva and gingival recession was considered, it was seen that with decrease in the adequacy of the attached gingiva, there was more gingival recession. Performing the tension test assessed the adequacy of attached gingiva. Stoner and Mazdyasna (10) reported similar findings stating that gingival recession had been caused by anatomical features of soft tissue eg. high frenum attachment associated with narrow band of attached gingiva which might cause excessive tension on the marginal tissue.

Another interesting observation was the relationship between malaligned teeth and gingival recession. It was found that $62.9 \%$ of labially placed teeth showed gingival recession which was much higher when it was compared to teeth which were correctly placed (37.04\%) or lingually placed teeth $(0.07 \%)$. These results were similar to the findings of Stoner and Mazdyasna (10) and Hirschfeld (15) who found that labially placed teeth had thin buccal plate or even absence of it when a tooth was tilted buccally.

It was interesting to find out from the above data that factors causing gingival recession were toothbrushing method, type of toothbrush, frequency of brushing, oral hygiene, gingival inflammation, tooth position, frenal pull and adequacy of attached gingiva, when considered in totality. Gingival recession was always the result of more than one factor acting together. The present study did not support any of the earlier beliefs of asymmetric recession, believed to be a result of right or left handed tooth brushing. Although this aspect was not considered separately during the study, in general the severity of gingival recession was almost symmetrical.
In addition there are probably many more implicating factors other than the ones already mentioned in this study in the initiation of gingival recession that may have not been considered here.

In shallow recession of grade 1 type of gingival recession, where adequate attached gingiva may be present, it could be best treated by a more conservative approach, which mainly consist of proper plaque control by means of scaling, root planning by the dentist as well as self homecare by the patient so as to prevent further progression of recession. Modification of brushing technique from horizontal to Bass method and the use of soft toothbrush rather than hard or medium toothbrush usually would suffice. Correction of the malaligned teeth is recommended and consultation with an orthodontist would be advisable. When recession is progressive, and in cases of grade II and grade III gingival recessions, in addition to conservative measures, orthodontic treatment and various soft tissue graft procedures may be needed to create a stable and healthy periodontium. In very severe cases like grade IV gingival recession with advanced destruction of the supporting structures where it is impossible to conserve it by any means, than it is best to advise extraction and then replacement with fixed or removable prosthodontics.

In conclusion, the information gathered would probably help in evolving long-term strategies to prevent occurrence of mucogingival defects and enable us to predict the rate of success of therapeutic measures. At the community level, adequate awareness and education in oral hygiene maintenance should prove to be fruitful in long run. The prevention of gingival recession should be the real goal rather than treating it once it has already occurred.

\section{BIBLIOGRAPHY}

1. Leo H. Natural History of Periodontal disease in man. J Periodontal 1992; 63: 489.

2. Smukler H, Landsberg J. The Toothbrush and gingival traumatic injury. J Periodontal 1984; 55 : 12 .

3. Miller P D Jr. A Classification of Marginal tissue Recession. J Periodontal Res Dent 1985; 5 : 9.

4. Tenebaum Henri. A clinical Study, Comparing the width of the attached gingiva and the prevalence of gingival recession. J Clin Periodont 1982; 9.

5. Leo H and Silness J. Periodontal disease in Pregnancy. Acta Odontol Scand 1963; 12: 533.

6. Placek $M$ and Skach M. Significance of labial frenum attachment in periodontal disease in Man Part I. Classification and epidemiology of the labial frenum attachment. J Periodontal 1974; 45: 12. 
7. Kisch T, Badersten A, Land Egelberg. Longitudinal Observation of unattached mobile gingival area. J Clin Periodontal 1986; 13: 131.

8. Buhl-S Does. Occlusal overload lead to gingival recession. ZWR 1991; Nov 100: 11.

9. Bernimoulin J, Curiloview Z. Gingival recession and tooth Mobility. J Clin Periodont 4; 2: 107 114.

10. Stoner E.J, Simon. M. Gingival Recession in the lower incisor. region of 15 year old subjects. J Periodont 1980; 51: 1.

11. Ainoma J, Palohiemo L. Gingival recession in school children at 7,12 and 17 years of age in Espoo Finland. Community Dent Oral Epidemio 1986; 15: 283-286.

12. Akpata E.S, Jackson D. Prevalence and distribution of gingivitis and gingival recession in children and young adults in Lagos, Nigeria. J Periodont 1979; 50(2): 79-83.
13. Kitchen PC. Prevalence of tooth root exposure and the relationship of the extent of such exposure to the degree of absorption in different age class. J Dent Res 1941; 20: 565.

14. Gorman W J. Prevalence and etiology of gingival recession. J Periodont 1967; 38: 316.

15. Hirschfeld I. The Toothbrush its use and abuse: Traumatization of the soft tissue by toothbrush. Dent Item Interest 1930; 56: 159.

16. Gillette WB, Vanhouse RL. Effect of Improper Oral procedures. J Am Dent Assoc 1980; 101: 3.

17. O'Leary TJ, Drake RB, Allern MF. The incidence of recession in young males. A further study. J Periodont 1971; 42: 264.

18. Parfitt G.J, Mjor I A. A Clinical Evaluation of local gingival recession in children. $\mathrm{J}$ Dent for Children. 1964; 31: 257-262.

19. Enslie R.D. Localized gingival recession. Int Dent J 1958; 8: 18 . 